\title{
A predicate calculus with control of derivations
}

\section{Report}

Author(s):

Mey, Daniel

Publication date:

1989

Permanent link:

https://doi.org/10.3929/ethz-a-000535756

Rights / license:

In Copyright - Non-Commercial Use Permitted

Originally published in:

ETH, Eidgenössische Technische Hochschule Zürich, Departement Informatik, Institut für Theoretische Informatik 120 


\section{ETH}

Eidgenössische

Technische Hochschule

Zürich
Departement Informatik Institut für

Theortische Informatik

Daniel Mey

\section{A Predicate Calculus with Control of Derivations}

December 1989 
Author's address:

Institut für Theoretische Informatik

ETH-Zentrum

$\mathrm{CH}-8092$ Zürich, Switzerland

e-mail: mey@inf.ethz.ch

(C) 1989 Departement Informatik, ETH Zürich 
Abstract A fragment of classical predicate calculus which does not contain rules for contraction is defined. It is shown to be decidable and yet propositionally complete. A semantics which reflects its constructive character is developed.

\section{Notions *}

For an introduction to mathematical logic, see e.g.

Y.I. MANIN. A coarse in mathematical logic. Springer (1977) or

E. MENDELSON. Introduction to mathematical logic. Van Nostrand (1964)

or (German)

H-D. EBBINGHAUS. Einführung in die mathematische Logik. Wissenschaftliche Buchgemeinschaft Darmstadt (1986).

A formal language expresses properties of mathematical objects. A language of first order predicate logic consists of an alphabet with symbols for (individual) variables, constants, functions and relations, logical connectives $(\neg$ for negation, $\wedge$ for conjunction, $\vee$ for disjunction, $\rightarrow$ for implication), universal $(\forall)$ and existential ( $\exists$ ) quantification over individual variables (in contrast to quantification over higher order variables) and auxiliary symbols (commas and parantheses). The formulas of the language are finite strings built up inductively from these symbols. The language of propositional logics usually contains propositional variables and symbols for the connectives only (hence no quantifiers!).

The semantics of a predicate logic specifies the meaning of the formulas by assigning properties of given mathematical entities. For the semantics of classical first order logic, these entities are mathematical structures consisting of a universe of individual objects, mathematical functions, and relations, corresponding to the language considered. A formula is valid in a structure if the assigned properties hold (e.g. $\forall x \exists y(y>x)$ is valid in the structure $N$

* This section is supplementary and not a part of the original paper. 
of natural numbers). A formula is valid if it is valid in every structure of the language considered.

A calculus for a logic consists of formal rules which can be combined in a defined way to form derivations (or formal proofs) for certain formulas which are called provable. A calculus is sound with respect to (wrt.) a semantics if every provable formula is valid wrt. the semantics; it is complete if every valid formula is provable. Sometimes, completeness can be established by showing that the Lindenbaum-algebra of the calculus (consisting of the classes of provably equivalent formulas) is a structure where the provable formulas are precisely the valid ones.

The rules of a sequent calculus are not directly concerned with formulas, but with sequents. Sequents are expressions of the form $\Gamma \supset \Pi$ where $\Gamma$ and $\Pi$ are finite sequences of formulas. Informally, $\Gamma \supset \Pi$ can be thought of the implication: conjunction of the formulas in $\Gamma$ implies disjunction of the formulas in $\Pi$. A rule consists of zero hypotheses (axioms), or one or more hypotheses - and a conclusion. A derivation is a tree with rules as nodes and axioms as leaves. The familiar Gentzen sequent calculus $\mathbf{L K}$ for classical predicate logic e.g. contains as axioms the sequents $E \supset E$ for prime formulas $E$ (formulas without connectives or quantifiers) and 19 other rules, one of them being the cut rule (see section 2) which can be eliminated from the calculus in the sense that every provable formula can be proved without the use of it (cut-elimination). The Gödel completeness theorem states soundness and completeness of LK wrt. the usual semantics.

Nevertheless, the set of these formulas is undecidable: there exists no decision procedure - an algorithm (or program), which determines whether or not an arbitrarily given formula is derivable. But there exist many mechanical methods which help finding proofs. Many of these methods rely on a unification process: two expressions containing unification variables are given and it is asked whether there exists a unifier. A unifier is a substitution containing expressions which, substituted for the variables of the given expressions, make them equal. A most general unifier is a unifier with the property that every other unifier can be obtained by another substitution. 


\section{Introduction}

Sometimes it is very hard to guess what a derivation of a formula of first order predicate calculus could look like. Church has shown in 1936 that the problem of deciding whether there is a derivation at all is undecidable (see e.g. [BJ]). Krajicek and Pudlak demonstrated in 1988 that deciding whether the formula has a proof with a given skeleton (only terms are not determined) is also undecidable [KP].

The formal reason for these phenomena is that calculi for classical predicate calculus always include rules which are contracting in the sense that a hypothesis can be shorter than a conclusion (measuring e.g. the number of logical symbols of the formulas). In Gentzen's familiar calculus $\mathbf{L K}$, these rules are the cut rule and the rules for contraction. If we drop them, we will demonstrate that we get a decidable system. The decision procedure basically evaluates all potential derivations and then computes whether a valid proof has been constructed. This is related to the procedure of determining membership of a word in a context sensitive language.

One modification of such a calculus, already being used as a core of a proof checking system, is studied by Ketonen and Weyhrauch [KW]. It proves the cut rule, but is even propositionally incomplete (e.g. $A \rightarrow A \wedge A$ is not derivable). In Girard's linear logic [Gi], the rules for weakening are also dropped, the only structural rules being those for exchange (see also e.g. [Av] and [GL]). In contrast, the calculus LS is designed for proving as many classically valid formulas as possible without including any contracting rules. In section 4 , it is shown to be propositionally complete. Formulas $B$ of the form $\exists^{*} C$ with $\exists^{*}$ a sequence of existential quantifiers and $C$ quantifier-free are classically provable iff there is a natural number $n$ for which the $n$ fold disjunction $\quad B \vee \ldots \vee B$ is LS-provable. This also holds for a calculus with further restrictions on the rules, having the additional property that in that case, there are terms $t_{1}, \ldots, t_{n}$ for which the disjunction $C t_{l} \vee \ldots \vee C t_{n}$ is provable. This fragment will be shown to be complete wrt. a semantics developed in section 4 . There, validity of a formula is not defined depending on an individual mathematical structure with functions, constants and relations; but depending on a class of structures having the same functions and constants. (A semantics for intuitionistic contraction-free logics is developed by Ono and Komori [OK].) 


\section{The calculus}

The calculus LS is a Gentzen sequent calculus and obtained from the classical LK by dropping the cut rule and the rules for contraction and by changing the $(\rightarrow$ le)-rule.

Def. LS is a Gentzen sequent calculus given by the following axioms and rules. The following notations are used:

capital Greek letters

capital Roman letters

$s, t, \ldots$

$u, v, \ldots$

$x, y, \ldots$

VC!

$\supset$ sequents of formulas

formulas ( $E$ prime)

terms

free variables

bounded variables

variable condition: eigenvariable $u$ not in lower sequent separation sign

AXIOMS

$E \supset E$

\section{RULES}

$\Gamma \supset \Pi$

$T, \Gamma \supset \Pi$

$\Gamma, T, Z, \Sigma \supset \Pi$

$\Gamma, Z, T, \Sigma \supset \Pi$

(Ele)

(W le)

$\Gamma \supset \Pi, T$

$\neg T, \Gamma \supset \Pi$

$(-1 \mathrm{l})$
$\Gamma \supset \Pi$

$\overline{\Gamma \supset \Pi, T}$

$\Gamma \supset \Pi, T, Z, \Omega$

$\Gamma \supset \Pi, Z, T, \Omega$

$T, \Gamma \supset \Pi$

$\overline{\Gamma \supset \Pi, \neg T}$
(W ri)

(Emin

$(\neg$ ri) 


$$
\frac{T, Z, \Gamma \supset \Pi}{T \wedge Z \Gamma \supset \Pi} \quad \text { (Ale) } \quad \frac{\Gamma \supset \Pi, T \quad \Gamma \supset \Pi, Z}{\Gamma \supset \Pi, T \wedge Z}
$$$$
\frac{T, \Gamma \supset \Pi \quad Z, \Gamma \supset \Pi}{T \vee Z, \Gamma \supset \Pi} \quad(\vee \mathrm{le}) \quad \frac{\Gamma \supset \Pi, T, Z}{\Gamma \supset \Pi, T \vee Z} \quad(\vee \text { ri })
$$$$
\frac{\Gamma \supset \Pi, T \quad Z, \Gamma \supset \Pi}{T \rightarrow Z, \Gamma \supset \Pi} \quad(\rightarrow \text { le }) \quad \frac{T, \Gamma \supset \Pi, Z}{\Gamma \supset \Pi, T \rightarrow Z} \quad(\rightarrow \mathrm{ri})
$$$$
\frac{F(t), \Gamma \supset \Pi}{\forall x F(x), \Gamma \supset \Pi} \quad(\forall \mathrm{le}) \quad \frac{\Gamma \supset \Pi, F(u)}{\Gamma \supset \Pi, \forall x F(x)} \mathrm{vC} \quad(\forall \mathrm{ri})
$$$$
\frac{F(u), \Gamma \supset \Pi}{\exists x F(x), \Gamma \supset \Pi} \text { vC! } \quad \underline{(\exists \mathrm{le})} \quad \frac{\Gamma \supset \Pi, F(t)}{\Gamma \supset \Pi, \exists x F(x)} \quad \underline{(\exists \mathrm{ri})}
$$

According to Girard's terminology, the formulations of the three rules with two hypotheses are called additive; the corresponding multiplicative formulations are as follows:

$$
\begin{aligned}
& \Gamma \supset \Pi, T \quad \Sigma \supset \Omega, Z \\
& \overline{\Gamma, \Sigma \supset \Pi, \Omega, T \wedge Z \quad(\wedge \text { rim) }} \\
& \frac{T, \Gamma \supset \Pi \quad Z, \Sigma \supset \Omega}{T \vee Z, \Gamma, \Sigma \supset \Pi, \Omega} \quad \underline{(\text { vle m) }} \\
& \Gamma \supset \Pi, T \quad Z, \Sigma \supset \Omega \\
& T \rightarrow Z, \Gamma, \Sigma \supset \Pi, \Omega
\end{aligned}
$$


Modifications of LS are obtained by replacing one or several of the additive formulations by multiplicative ones. In the presence of contraction, however, all these modifications are equivalent: a proof in one calculus can be transformed into a proof in the other by weakenings and contractions.

Below, two important properties of LS are proved.

PROPERTY 1. LS is properly weaker than LK.

PROOF. An LS-proof Q can be transformed into an LK-proof inductively on the depth of $\mathrm{Q}$ by inserting contractions after $(\rightarrow$ le)inferences. The converse is false: take e.g. $\exists x(R(a) \vee R(b) \rightarrow R(x))$ provable in LK but not in LS: The last rule applied in a proof would be $(\exists \mathrm{ri}$ ) with an upper sequent $\supset R(a) \vee R(b) \rightarrow R(t)$ and consequently both $R(a) \supset R(t)$ and $R(b) \supset R(t)$ would be provable for a term $t$, which is impossible.

PROPERTY 2. The cut rule

$$
\Gamma \rightarrow \Pi, A \quad A, \Sigma \rightarrow \Omega
$$

$$
\Gamma, \Sigma \rightarrow \Pi, \Omega
$$

is not valid in LS.

PROOF. Let $B: \exists x(R(a) \vee R(b) \rightarrow R(x))$ as in the proof above. $B \vee B$ is provable: $\supset B \vee B$ is provable $\Leftarrow$ $\supset R(a) \vee R(b) \rightarrow R(a), R(a) \vee R(b) \rightarrow R(b)$ is provable $\Leftarrow$ $R(a) \vee R(b), R(a) \vee R(b) \supset R(a), R(b)$ is provable $\Leftarrow$ $R(a) \vee R(b) \supset R(a), R(b)$ is provable, which is true.

Furthermore, $B \vee B \supset B$ is clearly provable. If the cut rule was valid, a proof of $B$ would follow, which was shown to be impossible in the proof above. 


\section{Decidability}

The decision procedure for LS described below is devided into two steps:

1. Evaluate all potential derivations of a given formula from bottom to the top. This is possible because the upper sequent(s) of an inference are in a certain sense shorter than the lower one(s). However, the terms in ( $\forall$ le) and $(\exists$ ri) have to be replaced by special variables.

2. Compute whether the potential derivations of step 1 contain a correct one: in order to do this, determine the terms in ( $\forall$ le) and $(\exists$ ri) by a unification process.

The following notation is used.

Def. LS' is obtained from LS by:

- dropping exchange-rules

- allowing arbritary permutations of formulas in the sequents of the rules.

REMARK. LS' and LS are equivalent: a proof in one of the calculi can be transformed into one in the other by inserting or deleting exchange-inferences.

Def. $\mathbf{L S}(\mathbf{b})$ is obtained from $\mathbf{L S}$ ' by the following:

- language extended with term-variables $a_{1}, \ldots, a_{b}$ and new free variables $v_{1}, \ldots, v_{b}$

- axioms have the form $R(\ldots) \supset R(\ldots)$

- eigenvariables at $\left(\forall\right.$ ri) and $\left(\exists\right.$ le) are among $v_{l}, \ldots, v_{b}$. No variable conditions!

- terms $t$ at $\left(\forall\right.$ le) and $\left(\exists\right.$ ri) are among $a_{1}, \ldots, a_{b}$.

LEMMA 1. Let $S$ be a sequent, b natural number. Then all LS(b)proofs of S can be computed.

PROOF. By induction on $\mathbf{n}(\mathbf{S})$, the number of logical symbols plus number of predicate signs in $S$.

$\mathrm{n}(\mathrm{S})=0$ The only possible proof of $\mathrm{S}$ is $\mathrm{S}$ itself (conclusions $\mathrm{C}$ of rules have $n(C)>0$ ). So just decide whether $S$ is an axiom.

$\mathrm{n}(S)>0$. Determine all possible hypotheses of inferences with 
conclusion S. Note that eigenvariables and terms of quantifierinferences have to be chosen among $v_{1}, \ldots, v_{b}$ and $a_{1}, \ldots, a_{b}$ respectively. Now, for each of the determined hypothesis $H$, $\mathrm{n}(\mathrm{H})<\mathrm{n}(\mathrm{C})$. Therefore, by induction, all $\mathbf{L S}(\mathbf{b})$-proofs of $\mathrm{H}$ are obtained. These proofs, combined with the corresponding inferences, yield all $\mathbf{L S}(\mathbf{b})$-proofs of S.

2ef. Let $\mathrm{P}^{*}$ be an $\mathrm{LS}(\mathrm{b})$-proof. The unification problem for $\mathrm{P}^{*}$ is the unification problem for the pairs of terms corresponding to each other in the axioms of $\mathrm{P}^{*}$, where the only variables of the problem are the term-variables.

THEOREM 1. The following algorithm decides LS:

Input: sequent $S$ with $n(S)=b$.

Output: an LS'-proof, if S is provable; else: message "not provable".

1. Compute all $\mathbf{L S}(\mathrm{b})$-proofs of $S$.

2. If there is a $\mathrm{P}^{*}$ computed in step 1 for which the unification problem is solvable by a most general unifier (mgu) $\sigma$ and for which $\mathrm{P}^{*} \sigma$ doesn't violate the variable conditions, return $\mathrm{P}^{*} \sigma$; else: message.

PROOF. termination Lemma 1 implies that step 1 is computable. For each P* computed in step 1 , the decisions of step 2 are computable (use Robinson-algorithm for deciding the unification problem).

correctness $P^{*} \sigma$ returned Because $\sigma$ is a unifier for the pairs of terms in the axioms of $\mathrm{P}^{*}$, the initial sequents of $\mathrm{P}^{*} \sigma$ are LS'-axioms. The inferences of $\mathrm{P}^{*}$ are transformed into inferences of $P^{*} \sigma$. Note that the variable conditions are satisfied. Finally, the last sequent of $P^{*} \sigma$ is $S$, because it does not contain any term-variable. Therfore, $\mathrm{P}^{*} \sigma$ is an LS'-proof of S and S is LS-provable.

"not provable" returned Suppose $S$ would be provable. It will be proved that no message would be returned, which is a contradiction.

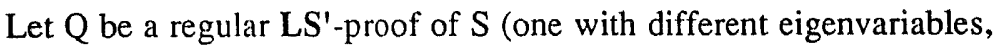
none of them contained in S. Such a proof can be easily obtained 
from an arbitrary one by a renaming of variables). Replace the eigenvariables by variables among $v_{1}, \ldots, v_{b}$, obtaining the LS' proof $\mathrm{P}$ of S. Then, replace in $\mathrm{P}$ the terms $t_{1}, \ldots, t_{b}$ of $(\forall 1 \mathrm{le})$ and $(\exists$ ri) at these inferences, and above them, by term-variables among $a_{1}, \ldots, a_{b}$, yielding an $\mathbf{L S}(\mathbf{b})$-proof $\mathrm{P}^{*}$ of $\mathrm{S}$. The unification problem of $\mathrm{P}^{*}$ is solvable, $\alpha=\left\{a_{1} / t_{1}, \ldots, a_{n} / t_{n}\right\}$ is a unifier. Let $\sigma$ be an $\mathrm{mgU}$. It remains to show that $\mathrm{P}^{*} \sigma$ satisfies the variable conditions like $\mathrm{P}^{*} \alpha=\mathrm{P}$ does. Let $\mathrm{H}^{*} / \mathrm{C}^{*}$ be a quantifier-inference of $\mathrm{P}^{*}$ with eigenvariable $w$. Suppose $C^{*} \sigma$ contains $w$. Let $\gamma$ be a substitution with $\alpha=\sigma \gamma$. Then $w$ is also contained in $C^{*} \alpha$. Therefore, $\quad H^{*} \alpha / C^{*} \alpha$ violates the variable condition of $\mathrm{P}^{*} \alpha$. Hence contradiction. So $\mathrm{P}^{*} \sigma$ satisfies the variable conditions and thus no message will be returned.

\section{REMARKS}

1. The above algorithm is not sensitive to additive or multiplicative formulations of the rules. Hence, it is a decision procedure for the modifications of LS as well.

2. The algorithm demonstrates that an LS-proof can contain sequents with many symbols although it proves a sequent with few symbols. Take e.g.

S: $\forall x \forall z R(x, f x x, z) \supset \exists y R(f c c, y, f y y)$ which has the following LS(b)-proof $\mathrm{P}^{*}$ :

$$
\begin{aligned}
& \frac{R\left(a_{1} f a_{1} a_{1}, a_{3}\right) \supset R\left(f c c, a_{2}, f a_{2} a_{2}\right)}{\forall z R\left(a_{1} f a_{1} a_{1}, z\right) \supset R\left(f c c, a_{2} f a_{2} a_{2}\right)} \\
& \hline \forall x \forall z R(x, f x x, z) \supset R\left(f c c, a_{2}, f a_{2} a_{2}\right) \\
& \hline \forall x \forall z R(x, f x x, z) \supset \exists y R(f c c, y, f y y)
\end{aligned}
$$

The unification problem is solved by the $\mathrm{mgU}$ $\sigma=\left\{a_{1} / f c c, a_{2} / f f c c f c c, a_{3} / f(f f c c f c c, f f c c f c c)\right\} . \mathrm{P}^{*} \sigma$ is an LS-proof of $\mathrm{S}$. S contains 18 symbols (commas and paranthesises not counted), but the first sequent contains 56 ! 


\section{Propositional part}

All rules for connectives of LS are reversible: the lower sequent is provable iff the upper one(s) is (are). This implies the provability of quantifier-free (qf.) contractions. As a consequence, the qf-parts of LK and LS are shown to be equivalent. This is used to establish a simple syntactic relation between the two calculi.

LEMMA 2. All LS-rules for connectives are reversible.

PROOF. The lemma will be shown for LS' (which is equivalent). Let $\mathrm{P}$ be a proof of the conclusion $\mathrm{C}$ of a rule for connectives. Proof by cases:

$(\neg-$ li) $\mathrm{C}: \neg B, \Delta \rightarrow \Lambda$. Proof by induction on the depth of P:

$\mathrm{d}(\mathrm{P})=0$ impossible.

$\mathrm{d}(\mathrm{P})>0$ Subcases:

$$
\frac{\Gamma \supset \Pi}{T, \Gamma \supset \Pi}
$$

(W le)

If $T$ is not $\neg B$, then apply the ind. hyp. and a (W le)-inference. If $T$ is $\neg B$, then replace the inference by a (W ri) with weakening formula $T$.

$$
\frac{\Gamma \rightarrow \Pi, T}{\neg T, \Gamma \rightarrow \Pi}
$$

If $\neg T$ is not $\neg B$, then apply the ind. hyp. and a $(\neg$ le)-inference. If $\neg T$ is $\neg B$, then drop the inference.

$$
\frac{\neg B, \Gamma \supset \Pi, T \quad \neg B, \Gamma \supset \Pi, Z}{\neg B, \Gamma \supset \Pi, T \wedge Z}
$$

$(A$ ri)

By induction proofs of $\Gamma \supset \Pi, T, B$ and $\Gamma \supset \Pi, Z$ are obtained. Now apply $(\wedge \mathrm{ri})$.

other subcases $\ldots$ by induction.

$(\neg \mathrm{ri}),(\wedge \mathrm{le}),(\wedge$ ri $),(\vee \mathrm{le}),(\vee \mathrm{ri}),(\rightarrow \mathrm{le}),(\rightarrow \mathrm{ri})$ similar. 
REMARK. Observe that the proofs of the hypotheses are obtained from the proofs of its conclusions without incrementing their depths.

LEMMA 3. For qf. formulas, (C le) and (C ri) are provable.

PROOF The lemma is proved for (C le); it is similar for (C ri). By induction on the depth of a proof $\mathrm{Q}$ of $B, B, \Delta \supset \Lambda$ it is shown that $B$, $\Delta \supset \Lambda$ is provable as well, provided $B$ is qf.

$\mathrm{d}(\mathrm{Q})=0$ impossible!

$\mathrm{d}(\mathrm{Q})>0$ cases:

$$
\frac{\Gamma \supset \Pi}{T, \Gamma \supset \Pi}
$$

(Wle)

If $T$ is not $B$, then apply the ind. hyp. and a (W le)-inference. If $T$ is $B$, then drop the inference.

$$
\frac{\Gamma \supset \Pi, T}{\neg T, \Gamma \supset \Pi}
$$

$\rightarrow$ le). $\rightarrow T$ is not $B$

Apply the ind. hyp. and a $(\neg$ le)-inference.

$$
\frac{\neg T, \Gamma \supset \Pi, T}{\neg T, \neg T, \Gamma \supset \Pi} \quad(\neg \mathrm{le}), \neg T \text { is } B
$$

Lemma 2 yields a proof of $\Gamma \supset \Pi, T, T$ with the same depth as the proof of

$\neg T, \Gamma \supset \Pi$, $T$. Now, applying the ind. hyp., a proof for $\Gamma \supset \Pi, T$ and with ( $\neg$ le) one for $\neg T, \Gamma \supset \Pi$ is obtained.

(other cases) similar!

THEOREM 2. The quantifier-free parts of $\mathbf{L S}$ and $\mathbf{L K}$ are equivalent.

PROOF. Property 1 shows that if a qf. formula $B$ is provable in LS, then also in LK. Lemma 3 now implies the converse: let $Q$ be a classical proof of $B$. Applying cut-elimination, $\mathrm{Q}$ can be assumed cutfree. An LS-proof of $B$ can be obtained from $Q$ by inserting 
weakenings at $(\rightarrow$ le $\mathrm{m}$ )-inferences and by applying lemma 3 at contractions.

REMARK. Theorem 2 does not hold for modifications of LS where one or more of the additive rules are replaced by their multiplicative formulations. If the modification contains $(\wedge \mathrm{ri})$, then it is easily verified that the classically valid formula $E \rightarrow E \wedge E$ is not provable. If it contains ( $\vee$ le), then $E \vee E \rightarrow E$ is not provable. Finally, if it contains $(\rightarrow$ le), then $(\neg E \rightarrow E) \rightarrow E$ is not provable.

For formulas of the form $\exists * C$ with $\exists *$ a sequence of existential quantifiers and $C$ quantifier-free, theorem 2 can be used to formulate a simple syntactic relation between the two calculi. Because every formula is classically equivalent to a formula in the above form, its classical provability can be reduced to a provability-problem in LS.

COROLlaRY 1 . Let $B$ be of the form $\exists * C$ with $\exists *$ a sequence of existential quantifiers and $C$ quantifier-free. Then $B$ is LK-provable iff there is a natural number $n$ for which the $n$-fold disjunction $B \vee \ldots \vee B$ is LS-provable.

PROOF If $B \vee \ldots \vee B$ is LS-provable, then, by reversibility of ( $\vee$ re), $\supset B, \ldots, B$ is provable in $\mathbf{L S}$ and therefore in $\mathbf{L K}$. Now, apply contractions and obtain a proof of $B$.

For the converse, take an LK-proof of $B$ in Gentzen normal form (containing a qf. "midsequent" with no propositional inferences below it). The midsequent of this proof must have the form $\supset C\left(t_{1}\right), \ldots, C\left(t_{n}\right)$ for tuples of terms $t_{1}, \ldots, t_{n}$. Because of the propositional completeness of LS, this sequent is also LS-provable. $(\exists$ ri)- and possibly exchange-inferences now provide an LS-proof of the $n$-fold disjunction $B \vee \ldots \vee B$. 


\section{Semantics for a fragment}

The cut rule is not valid in LS (see section 2). Related to that, $\cong$ : "LS-provable equivalent" is not transitive and therefore not an equivalence relation. E.g. if $B$ is

$\exists x(R a \vee R b \rightarrow R x)$ then $R c \vee \neg R c \cong B \vee B . B \vee B \cong B$ as well, but $R c \vee \neg R c \cong B$ is false as can easily be seen. Therefore, we can't construct a Lindenbaum-algebra for LS, which could serve as a basis for an algebraic semantics.

The main motivation behind developing a semantics for a fragment of the calculus is not to have a tool for demonstrating a formula to be unprovable (this can be done without semantics because the calculus was shown to be decidable), but to give an example for a semantics which is propositionally complete and yet violates the cut rule.

The semantics described below reflects the constructive character of the calculus: if $\exists x B x$ is LS-provable, then there is a term $t$ such that $B t$ is provable. However, if $\Gamma \supset \Pi, \exists x B x$ is provable, one can not always find a $t$ such that $\Gamma \supset \Pi, B t$ is provable. As an example, $R a \vee R b \supset \exists x B x$ is provable, but $R a \vee R b \supset B t$ is not provable for any term $t$. We therefore modify LS appropriately:

Def. $\mathbf{L S}_{\mathbf{r}}: \mathbf{L S}$ but

- no $(\forall$ ri) or $(\exists \mathrm{le})$

- rules with two hypotheses: all formulas except principal formula are quantifier-free

- in sequents, each quantifier bounds a different variable.

$\mathbf{L S}_{\mathbf{r}}{ }^{\prime}$ is obtained from $\mathbf{L S}_{\mathbf{r}}$ as $\mathbf{L S} \mathbf{S}^{\prime}$ is defined from $\mathbf{L S}$.

Validity of formulas are not defined wrt. a single mathematical structure, but wrt. a class of structures which is determined by a universe, functions and constants corresponding to the language $L$ considered.

Def. $L:\left\{\mathrm{f}_{\mathrm{i}}\right\},\left\{\mathrm{c}_{\mathrm{j}}\right\},\left\{\mathrm{R}_{\mathrm{k}}\right\}$ first order language.

$\boldsymbol{L}$-class $\underline{\mathrm{A}}\left\{A \mid A L\right.$-structure with $\left.|A|=\mathrm{A}, \mathrm{f}_{\mathrm{i}} A=\mathrm{f}_{\mathrm{i}} \mathrm{A}, \mathrm{c}_{\mathrm{j}} A=\mathrm{c}_{\mathrm{j}} \mathrm{A}\right\}$ with $A \neq \varnothing,\left\{f_{i} A\right\},\left\{c_{j} A\right\}$ functions and constants corresponding to $L$.

$L(e): L$ plus e-variables: new free variables $e_{1}, e_{2}, \ldots$

w: assignment of free variables of $L$ 
o: assignment of e-variables ("e-assignment").

$B L(e)$-formula, $A \in \underline{\mathrm{A}}, \mathrm{w}, \mathrm{o}$ assignments of free and e-variables. $[B]_{\mathrm{W}, 0, A}$ defined inductively:

i) $[E]_{\mathrm{W}, \mathrm{o}},[-C]_{\mathrm{W}, \mathrm{o}},[\mathrm{C} * \mathrm{D}]_{\mathrm{W}, \mathrm{o}}(*$ is one of $\wedge, \vee$ or $\rightarrow)$ defined classically

ii) $\left[Q x_{k} C\left(x_{k}\right)\right]_{\mathrm{w}, 0}=\left[C\left(e_{k}\right)\right]_{\mathrm{w}, 0}$ where $\mathrm{Q}$ is $\forall$ or $\exists$

iii) $\left[A_{1}, \ldots A_{n} \supset B_{1}, \ldots, B_{m}\right]_{\mathrm{w}, \mathrm{o}}=\left[\neg A_{1} \vee \ldots \vee B_{1} \vee \ldots\right]_{\mathrm{w}, \mathrm{o}}$ (defined classically).

$B$ valid in $\underline{\mathrm{A}}$ (at w): exists o such that for all $A \in \underline{\mathrm{A}}:[B]_{\mathrm{w}, \mathrm{o}, A}=$ true $B$ e-valid: $B \underline{\mathrm{A}}$-valid in every $L$-class $\underline{\mathrm{A}}$ at every assignment $\mathrm{w}$.

Motivation. An $L$-class determines all objects and operations of a mathematical structure, but not the properties of these. A formula is true in the class at an assignment iff there is an e-assignment such that the formula with deleted quantifiers is (classically) true in all structures of the class, provided the bounded variables are assigned to the corresponding components of $o$. (o can be regarded as "oracle" for the bounded variables, making the formula true however the predicates are interpreted.) If $B$ is an existential formula where each quantifier bounds a different variable, then the definition becomes classical, if in the definition of validity in a class, "exists 0 " and "for all $A^{\prime \prime}$ are changed.

EXAMPLES $B_{k}: \exists x_{k}\left(R(a) \vee R(b) \rightarrow R\left(x_{k}\right)\right)$

1. $B_{k}$ is not e-valid:

Let $\underline{A}$ be such that $a^{A} \neq b^{A}, 0$ arbitrary. Then either $a^{A}$ or $b^{A}$ is different from $o_{k}$. Assume without loss of generality that $o_{k} \neq a A$. Let $A \in \underline{\mathrm{A}}$ be such that $\mathrm{a}^{\mathrm{A}} \in \mathrm{R} A$ but not $\mathrm{o}_{\mathrm{k}} \in \mathrm{R} A$. This implies $\left[B_{k}\right]=\left[R(a) \vee R(b) \rightarrow R\left(e_{k}\right)\right]=$ false. Hence, the formula is not valid in $\underline{\mathrm{A}}$ and thus not e-valid.

2. If $\mathrm{A}$ has one element, then $B_{k}$ is valid in $\underline{A}$ :

Because $A$ has just one element $\mathbf{c}$, o must be a constant assignment. Furthermore, $\mathrm{a}^{\mathrm{A}}=\mathrm{b}^{\mathrm{A}}=\mathrm{c}$. Then $\left[B_{k}\right]_{\mathrm{w}, \mathrm{o}}=\left[R(a) \vee R(b) \rightarrow R\left(e_{k}\right)\right]_{\mathrm{w}, \mathrm{o}}=$ true and the formula is valid in $\underline{A}$.

3. $B_{1} \vee B_{2}$ is e-valid: 
Let $\underline{\mathrm{A}}$ be a class. Choose $\mathrm{o}_{1}=\mathrm{a} \mathrm{A}, \mathrm{o}_{2}=\mathrm{b} \mathrm{A}$. By definition , $\left[B_{1} \vee B_{2}\right]_{\mathrm{w}, \mathrm{o}}=$ $\left[B_{1}\right]_{\mathrm{w}, \mathrm{o}} \vee\left[B_{2}\right]_{\mathrm{w}, \mathrm{o}}=\left[R(a) \vee R(b) \rightarrow R\left(e_{1}\right)\right]_{\mathrm{w}, \mathrm{o}} \vee\left[R(a) \vee R(b) \rightarrow R\left(e_{2}\right)\right]_{\mathrm{w}, \mathrm{o}}$. If neither $\mathrm{a}^{\mathrm{A}}$ nor $\mathrm{b}^{\mathrm{A}}$ are in $\mathrm{R}^{A}$, then both parts of the disjunction are true. If $\mathrm{a}^{\mathrm{A}}$ is in $\mathrm{R}^{A}$, then the left part is true. Finally, $\mathrm{f} \mathrm{b}^{\mathrm{A}}$ is in $\mathrm{R}^{A}$ then the right part is true.

4. $R(a) \vee R(b) \rightarrow \exists x_{k} R\left(x_{k}\right)$ is not e-valid:

Let $\underline{\mathrm{A}}$ be the class of example 1. Then $\left[R(a) \vee R(b) \rightarrow \exists x_{k} R\left(x_{k}\right)\right]=$ $[R(a) \vee R(b)] \rightarrow\left[R\left(e_{k}\right)\right]=\left[R(a) \vee R(b) \rightarrow R\left(e_{k}\right)\right]=$ false according to example 1 .

Below we will prove that wrt. e-validity, $\mathbf{L S}_{\mathbf{r}}$ is sound and complete. Showing completeness is much easier than showing soundness: If a sequent is valid, then it is valid in the so called canonical class under the canonical assignment described below. From this, a proof of the sequent may be obtained directly . For soundness, the quantifier inferences of the given proof must be encoded in a certain way.

LEMMA 4. $B L$-formula. If assignements $\mathrm{w}, \mathrm{w}^{\prime}$ agree on the components corresponding to free variables of $B$ and if e-assignments $0,0^{\prime}$ agree on the components corresponding to the bounded variables of $B$, then $[B]_{\mathrm{w}, \mathrm{o}}=[B]_{\mathrm{w}^{\prime}, \mathrm{o}^{\prime}}$.

PROOF By induction on $B$, the lemma is proven for $B$ an $L(e)$ formula with the additional assumption that $0,0^{\prime}$ also agree on the corresponding e-variables.

$B$ prime $\quad \mathrm{w}, \mathrm{w}^{\prime}$ and $0, \mathrm{o}^{\prime}$ agree on the (free) variables of $B$ which immediately implies the statement.

$B: \exists x_{k} C\left(x_{k}\right)\left[\exists x_{k} C\left(x_{k}\right)\right]_{\mathrm{w}, \mathrm{o}}=\left[C\left(e_{k}\right)\right]_{\mathrm{w}, \mathrm{o}}=$ (ind. hyp.) $\left[C\left(e_{k}\right)\right]_{\mathrm{w}^{\prime}, \mathrm{o}^{\prime}}=$ $\left[\exists x_{k} C\left(x_{k}\right)\right]_{w^{\prime}, o^{\prime}}$.

$B: \forall x_{k} C\left(x_{k}\right)$ identically!

other cases ... with induction hypothesis.

LEMMA 5. S $L$-sequent where each quantifier bounds a different variable. If $S$ is an axiom or a conclusion of an $\mathbf{L S}_{\mathbf{r}}{ }^{\prime}$-inference with 
hypotheses valid in $\underline{A}$, then $S$ is valid in $\underline{A}$.

PROOF. Let $\underline{A}$ be a class and $w$ an assignment. Cases:

$\mathrm{S}$ an axiom Then $\mathrm{S}$ is $E \supset E$. Choose o arbitrarily. Let $A$ be in $\underline{\mathrm{A}}$.

Then $[E \supset E]_{\mathrm{W}, \mathrm{o}}=[\neg E \vee E]_{\mathrm{W}, \mathrm{o}}=$ true.

other cases The hypotheses $\mathrm{H}^{1}$ (and $\mathrm{H}^{2}$ ) of the inference are valid in $\underline{\mathrm{A}}$. There exist $\mathrm{o}^{1}$ (and $\mathrm{o}^{2}$ ) such that for all $A \in \underline{\mathrm{A}}:[\mathrm{H}]_{\mathrm{w}, \mathrm{o}^{1}, A}=$ true (and $\left[\mathrm{H}^{\prime}\right]_{\mathrm{w}, \mathrm{o}^{2}, A}=$ true). Subcases:

$$
\begin{gathered}
\frac{\Gamma \supset \Pi}{T, \Gamma \supset \Pi} \\
{[T, \Gamma \supset \Pi]_{\mathrm{w}, \mathrm{o}}=[\neg T]_{\mathrm{w}, \mathrm{o}} \vee[\Gamma \supset \Pi]_{\mathrm{w}, \mathrm{o}}=\text { true for } \mathrm{o}=\mathrm{o}^{1}}
\end{gathered}
$$

(W ri) analogous.

$$
\begin{aligned}
& \Gamma \supset \Pi, T \\
& \neg T, \Gamma \supset \Pi \\
& {[\neg T, \Gamma \supset \Pi]_{\mathrm{w}, \mathrm{o}}=[\neg \neg T]_{\mathrm{w}, \mathrm{o}} \vee[\supset \Pi]_{\mathrm{w}, \mathrm{o}}=[T]_{\mathrm{w}, \mathrm{o}} \vee[\Gamma \supset \Pi]_{\mathrm{w}, \mathrm{o}}=[\mathrm{H}]_{\mathrm{w}, \mathrm{o}}=}
\end{aligned}
$$
true for $0=0^{1}$.

$\rightarrow$ ri) analogous.

$$
\begin{aligned}
& \frac{T, Z, \Gamma \supset \Pi}{T \wedge Z, \Gamma \supset \Pi} \quad \text { (^le) } \text { clear. } \\
& \frac{\Gamma \supset \Pi, T \quad \Gamma \supset \Pi, Z}{\Gamma \supset \Pi, T \wedge Z} \text { (^ri) }
\end{aligned}
$$

$o$ is obtained from $o^{1}$ by replacing the components corresponding to bounded variables of $Z$ by the same components of $o^{2}$. Because $\Gamma \supset \Pi$ is quantifier-free, lemma 4 implies that $[\Gamma \supset \Pi]_{\mathrm{w}, \mathrm{o}}=[\Gamma \supset \Pi]_{\mathrm{w}, \mathrm{o}}{ }^{1=}$ $[\Gamma \supset \Pi]_{\mathrm{w}, \mathrm{o}^{2}}$. Because each quantifier bounds a different variable, the bounded variables of $Z$ and $T$ are different. Lemma 4 therefore implies $[T]_{\mathrm{W}, \mathrm{o}}=[T]_{\mathrm{W}, \mathrm{o}^{1}}$. Finally, because $o$ and $\mathrm{o}^{2}$ agree on the components corresponding to the bounded variables of $Z$, lemma 4 implies $[Z]_{\mathrm{w}, \mathrm{o}}=[Z]_{\mathrm{w}, \mathrm{o}}$. Together, $[\Gamma \supset \Pi, T \wedge Z]_{\mathrm{w}, \mathrm{o}}=[\Gamma \supset \Pi, T]_{\mathrm{w}, \mathrm{o}} \wedge$ $[\Gamma \supset \Pi, Z]_{\mathrm{w}, 0}=[\Gamma \supset \Pi, T]_{\mathrm{w}, \mathrm{o}^{1}} \wedge[\Gamma \supset \Pi, Z]_{\mathrm{w}, \mathrm{o}^{2}}=$ true. 


$$
\frac{F(t), \Gamma \supset \Pi}{\forall x_{k} F\left(x_{k}\right), \Gamma \supset \Pi} \quad(\forall \text { le })
$$

Let $\mathrm{o}=\mathrm{o}^{1}\left[\mathrm{e}_{\mathrm{k}} /[\mathrm{t}]_{\mathrm{w}}\right]$. Then, $\left[\neg \forall x_{k} F\left(x_{k}\right)\right]_{\mathrm{w}, \mathrm{o}}=\left[\neg F\left(e_{k}\right)\right]_{\mathrm{w}, \mathrm{o}}=[\neg F(t)]_{\mathrm{w}, \mathrm{o}}$. Because neither $\neg F(t)$ nor $\Gamma \supset \Pi$ contains $x_{k}$, lemma 4 implies $[\neg F(t)]_{\mathrm{W}, \mathrm{o}}=[\neg F(t)]_{\mathrm{W}, \mathrm{o}}{ }^{1}$ and $[\Gamma \supset \Pi]_{\mathrm{W}, \mathrm{o}}=[\Gamma \supset \Pi]_{\mathrm{W}, \mathrm{o}}{ }^{1}$. Therefore, $\left[\forall x_{k} F\left(x_{k}\right), \Gamma \supset \Pi\right]_{\mathrm{w}, \mathrm{o}}=\left[\neg \forall x_{k} F\left(x_{k}\right)\right]_{\mathrm{w}, \mathrm{o}} \vee[\Gamma \Pi]_{\mathrm{w}, \mathrm{o}}=$ $[\neg F(t)]_{\mathrm{W}, \mathrm{o}}{ }^{1 \vee}[\Gamma \supset \Pi]_{\mathrm{W}, \mathrm{o}}{ }^{1}=[\mathrm{H}]_{\mathrm{w}, \mathrm{o}}{ }^{1}=$ true.

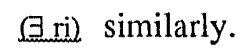

THEOREM 3. (soundness) $S L$-sequent where each quantifier bounds a different variable. If $\mathbf{S}$ is $\mathbf{L S}_{\mathbf{r}}{ }^{\prime}$-provable, then it is e-valid.

PROOF. By induction on $Q$ where $Q$ is a proof of $S$. $\mathrm{d}(\mathrm{Q})=0 \mathrm{~S}$ is an axiom. Lemma 5 implies it is e-valid. $d(Q)>0 \quad S$ is conclusion of an inference. Its upper sequent(s) are provable. The ind. hyp. implies e-validity of them, lemma 5 implies e-validity of the conclusion.

Def. i) $\mathbf{A}_{\text {kan }}$ canonical $L$-class: $\mathrm{A}_{\mathrm{k}}: L$-terms, $\mathrm{f}_{\mathrm{i}} \mathrm{A}, \mathrm{c}_{\mathrm{j}} \mathrm{A}$ canonical. ii) $w_{k a n}$ canonical l-assignment: $w_{k a n}(u)=u$.

LEMMA 6. If the conclusion of an $\mathbf{L S}_{\mathbf{r}}$ '-inference (not a weakening) is valid in $\underline{A}_{k a n}$ at $w_{k a n}$, then its hypotheses are also valid for appropriate terms if the inference is $(\forall$ le) or $(\exists$ ri).

PROOF. Cases:

$(\neg$ le $),(\neg \mathrm{ri}),(\wedge \mathrm{le}),(\vee \mathrm{ri}),(\rightarrow \mathrm{ri})$ compare corresponding cases of lemma $5:[\mathrm{C}]_{\mathrm{w}, \mathrm{o}}=[\mathrm{H}]_{\mathrm{w}, \mathrm{o}}$ for arbitrary $\mathrm{w}, \mathrm{o}$.

$$
\frac{\Gamma \supset \Pi, T \quad \Gamma \supset \Pi, Z}{\Gamma \supset \Pi, T \wedge Z}
$$

$[\Gamma \supset \Pi, T \wedge Z]_{\mathrm{w}, \mathrm{o}}=[\Gamma \supset \Pi, T]_{\mathrm{w}, \mathrm{o}} \wedge[\Gamma \supset \Pi, Z]_{\mathrm{w}, \mathrm{o}}$ for arbitrary $\mathrm{w}$ and $o$. 


$$
\frac{\Gamma \supset \Pi, F(t)}{\Gamma \supset \Pi, \exists x_{k} F\left(x_{k}\right)} \quad(\exists \mathrm{ri})
$$

$\mathrm{w}=\mathrm{w}_{\text {kan }}[\Gamma \supset \Pi, \exists x F(x)]_{\mathrm{w}, \mathrm{o}}=[\Gamma \supset \Pi]_{\mathrm{w}, \mathrm{o}} \vee\left[F\left(e_{k}\right)\right]_{\mathrm{w}, \mathrm{o}}$. Because $\mathrm{w}$ is canonical, $\left[F\left(e_{k}\right)\right]_{\mathrm{w}, 0}=[F(\mathrm{t})]_{\mathrm{w}, \mathrm{o}}$ where $\mathrm{t}=\mathrm{o}\left(\mathrm{e}_{\mathrm{k}}\right)$ is a term. Therefore the hypothesis of the inference is valid for an appropriate term in $\underline{A}_{\text {kan_at }}$ $w_{k a n}$, provided the conclusion is.

$(\forall$ le $)$ analogously.

THEOREM 4. (completeness) $L$-sequent $S$ is valid in $\underline{A}_{k a n}$ at $w_{k a n}$ iff it is $\mathbf{L S}_{\mathbf{r}} \mathbf{r}^{- \text {-provable. }}$

PROOF. $\leftleftarrows$ Theorem 3 .

symbols in $\mathrm{S}$.

$\Rightarrow$ By induction on IS where ISI is number of logical $\mathrm{n}=0 \mathrm{~S}: A_{1}, \ldots A_{n} \supset B_{1}, \ldots, B_{m}$ only consists of prime formulas. If every $A_{i}$ would be different from every $B_{j}$, then an $A \in \underline{A}_{\text {kan }}$ could be constructed which would make all $A_{i}$ 's true and all $B_{j}$ 's false. Therefore, $[S]_{A}=$ false and $S$ would not be valid in $\underline{A}_{k a n}$ at wkan. It follows that an $A_{i}$ and $B_{j}$ are identical. Hence, $\mathrm{S}$ can be proven with an axiom followed by weakenings.

$n>0 \mathrm{~S}$ is conclusion of an inference except weakening with hypotheses $\mathrm{H}_{1}$ (and $\mathrm{H}_{2}$ ).

Lemma 6 implies that they are valid in $\underline{A}_{k a n}$ at $w_{k a n}$. Because they have at least one logical symbol less than $S$, the induction hypothesis provides proofs for them which can be extended to a proof of $S$ by combining them with the inference.

COROLLARY 2 Sequents are e-valid iff they are $\mathbf{L S}_{\mathbf{r}}$-provable.

PROOF Because $\mathbf{L S}_{\mathbf{r}}$ and $\mathbf{L S}_{\mathbf{r}}{ }^{\prime}$ are equivalent, it suffices to prove that a sequent $S$ is e-valid iff it is $\mathbf{L S}_{\mathbf{r}}$-provable. Let $S$ be e-valid. Then $S$ is valid in $\underline{A}_{k a n}$ at $w_{k a n}$. Theorem 4 implies the provability of $S$. If $S$ is provable, then there exists a proof where every quantifier of a sequent bounds a different variable (rename the bounded variables of the proof). Theorem 3 now implies the validity of $S$. 
REMARK Example 4 above demonstrated that $R(a) \vee R(b) \rightarrow \exists x_{k} R\left(x_{k}\right)$ is not e-valid. Soundness of $\mathbf{L S}_{\mathbf{r}}$ implies that the formula is not $\mathbf{L S}_{\mathbf{r}}$ provable. Nevertheless, as mentioned before, the formula is provable in LS. But the proof uses an $(v$ le)-inference with upper sequents $R(a) \rightarrow \exists x_{k} R\left(x_{k}\right)$ and $R(b) \rightarrow \exists x_{k} R\left(x_{k}\right)$, thereby violating the $\mathbf{L S}_{\mathbf{r}^{-}}$ condition for the rules with two hypotheses.

\section{References}

[B] G. BOOlOS AND R. JefFrey. Computability and logic. Cambridge university press (1974)

[KP] J. KRAJICEK AND P. PUDLAK. The number of proof lines and the size of proofs in the first order logic. Archive for mathematical logic 27 (1988) 69 - 84

[KW] J. Ketonen, R. Weyhrauch. A decidable fragment of predicate calculus. Theoretical computer science (TCS) 32 (1984) 297 - 307

[Gi] J-Ý. GIRARD. Linear logic. TCS 50 (1987) 1 - 101

[GL] J-Y. GIRARD, Y. Lafont. Linear logic and lazy computation. Proceedings theory and practice of software development 1987 ( $\mathrm{H}$. Ehrig et al., editors). Lecture notes in computer science 250 (1987) 52 - 66

[AV] A. AVRON. The semantics and proof theory of linear logic. TCS 57 (1988) $161-184$

[OK] H. ONO AND Y. KOMORI. Predicate logic without the structure rules. Studia logica 45 (1985) 393 -404. 
Gelbe Berichte des Departements Informatik

109 H. Mössenböck Object Oberon: An object-Oriented Extension of

J. Templ Oberon

R. Griesemer

110 Ch. Vetterli

OPUS: Ein objektorientiertes Electronic Publishing System

111 N. Wirth

From Modula to Oberon and the Programming Language Oberon

112 N. Wirth

Ceres-Net: A Low-Cost Computer Network Extending Ceres-Net by a Mail Service

113 P. Schäuble

On the Compatibility between Retrieval Functions, Preference Relations, and Document Descriptions

114 M. Müller

CeNet: AppleTalk - kompatible KommunikationsSoftware für CERES

115 W. Gander

Solving Linear Equations by Extrapolation

G.H. Golub

D. Gruntz

116 B. Sanders

117 N. Wirth

Stepwise Refinement of Mixed Specifications of Concurrent Programs

Modula-2 and Object-Oriented Programming. Drawing Lines, Circles, and Ellipsis in a Raster. Flintstone.

118 H.-J. Schek

H.-B Paul

M.H. Scholl

The DASDBS Project: Objectives, Experiences, and Future Prospects

G. Weikum

119 J. Gutknecht

The Oberon Guide

120 D.Mey

A Predicate Calculus with Control of Derivations 